\title{
Multitap microwave photonic filters with programmable phase response via optical frequency comb shaping
}

\author{
Minhyup Song, ${ }^{1, *}$ Victor Torres-Company, ${ }^{1,2}$ Andrew J. Metcalf, ${ }^{1}$ and Andrew M. Weiner ${ }^{1}$ \\ ${ }^{1}$ School of Electrical and Computer Engineering, Purdue University, 465 Northwestern Avenue, West Lafayette, Indiana 47907, USA \\ ${ }^{2}$ Departament de Física, Universitat Jaume I, Castelló de la Plana, Castelló 12081, Spain \\ *Corresponding author: song55@purdue.edu
}

Received December 13, 2011; revised January 15, 2012; accepted January 15, 2012; posted January 17, 2012 (Doc. ID 159796); published February 22, 2012

\begin{abstract}
We present a programmable multitap microwave photonic filter with an arbitrary phase response operating over a broad bandwidth. Complex coefficient taps are achieved by optical line-by-line pulse shaping on a $10 \mathrm{GHz}$ flat optical frequency comb using a novel interferometric scheme. Through high-speed real-time measurements, we demonstrate programmable chirp control of a waveform via phase filtering. This achievement enables us to compress broadband microwave signals to their corresponding bandwidth-limited pulse duration. @ 2012 Optical Society of America

OCIS codes: $\quad 060.5625,070.2615,320.1590,320.5540$.
\end{abstract}

Over the past few decades, the field of microwave photonics has been investigated to enhance the performance of radio-frequency (RF) systems [1,2]. This perspective offers benefits such as ultrabroad bandwidth, insensitivity to electromagnetic interference, optical fiber transport, easy tuning, and programmability, which are features difficult or impossible to attain with purely electronic solutions. An important application of microwave photonics is the implementation of filters for high-frequency carrier, broad bandwidth RF waveforms [2]. The realization of programmable arbitrary $\mathrm{RF}$ phase control is a more challenging task, yet with promising implications in modern radar systems [3] or compensation of antenna distortions [4]. A scheme for microwave photonic filters (MWPFs) with programmable phase profiles, consisting of a hyperfine-resolution optical pulse shaper in an optical-to-electrical mapping configuration, is reported in [5]. The careful phase control allows for matched filtering of large time-bandwidth-product (TBWP) ultrawideband waveforms [6,7]. However, unlike with multitap dispersive filter schemes, here the longest time aperture of the waveform that can be processed is limited to $<1 \mathrm{~ns}$, owing to the resolution of the pulse shaper. In order to overcome the temporal aperture barrier, alternative processing schemes must be considered.

Conventionally, MWPFs are based on a delay-line multitap geometry, inspired by finite-impulse-response (FIR) digital filter design, featuring taps with positive- and negative-valued coefficients [2]. On the other hand, the implementation of complex-coefficient-tap FIR filters would enable new signal processing applications in radar, ultrawideband communication, and arbitrary waveform generation [8]. Recent research efforts to implement MWPFs with complex-coefficient taps include stimulated Brillouin scattering [9], a spatial light modulator used in a cross-polarized carrier-sideband geometry [10], specially designed fiber Bragg gratings [11], and a pulse shaper capable of resolving and applying different phases to optical carriers and sidebands [12]. However, these solutions are difficult to extend to a large number of taps, which prevents their use for applications that demand high selectivity and sharp control of the filter characteristics. In order to design MWPFs with a high number of complex taps, our group has adopted a dispersive delay-line scheme involving line-by-line pulse shaping of an optical frequency comb in an interferometer [13]. Synthesis of tunable and reconfigurable MWPFs with flat-top amplitude profile and high side-lobe suppression has been reported recently using such an approach [14]. In this Letter, we extend this concept to demonstrate programmable MWPFs with arbitrary phase response. In this geometry, the filter's time aperture and TBWP are governed by the total tap delay and number of comb lines, respectively. Given that Fourier processing of $>100$ comb lines is possible [15], this scheme constitutes an ideal platform to process broad-bandwidth microwave signals with increased temporal apertures and dispersion. Even more, unlike with other recently reported FIR dispersivedelay-line schemes [16,17], our setup simultaneously enables bandpass tuning $[\underline{14}]$ and phase reconfiguration in a convenient manner.

Figure 1 shows our MWPF scheme featuring multiple complex-coefficient taps. The optical frequency comb is implemented by cascading a properly biased intensity modulator (EOM) and an electro-optic phase modulator [18] driven by a $9.95 \mathrm{GHz}$ clock source, leading to a flat envelope with 21 lines at $-10 \mathrm{~dB}$ bandwidth. The comb is equally split into two paths after amplification with an EDFA. The comb through path 1 is single-sideband modulated in a dual-drive Mach-Zehnder modulator biased at the quadrature point and driven by a pair of copies

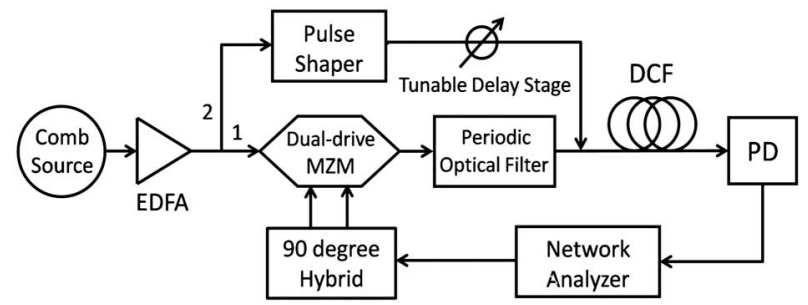

Fig. 1. MWPF architecture to implement complex tap weights. 
with $90^{\circ}$ phase difference of the $\mathrm{RF}$ signal to be processed. In order to suppress the comb carriers, the modulator output is sent through a periodic filter implemented with a delay-line interferometer (a commercial differential phase shift keying demodulator), which has deep nulls in its transmission frequency response with a periodicity equal to the repetition rate of the comb. The portion of the optical comb in path 2 is shaped by a pulse shaper (Finisar WaveShaper 1000S), which enables us to program the amplitude and phase of the individual comb lines. The two paths are subsequently aligned in polarization and coupled back with a 50/50 coupler. The light is sent to a dispersion compensating fiber (DCF) that has $-1259.54 \mathrm{ps} / \mathrm{nm}$ dispersion at $1550 \mathrm{~nm}$, resulting in a tap delay of $96 \mathrm{ps}$. The optical signal is detected by a $22 \mathrm{GHz}$ bandwidth photodiode (PD), and the amplitude of the transfer function (S21 parameter) is measured by a network analyzer. Physically, the output electrical signal is composed by a sum of beating terms between each of the shaped comb lines from path 2 and their corresponding adjacent RF sidebands from path 1 . In this way, optical line-by-line shaping in amplitude and phase enables the full complex control of the output RF signal.

The filter transfer function can be written as [14]

$$
H\left(\omega_{\mathrm{RF}}\right) \propto \sum_{n} e_{n}^{2} \exp \left[j n D \Delta \omega\left(\omega_{\mathrm{RF}}+\tau / D\right)-j \Phi_{n}\right],
$$

where $e_{n}^{2}$ is the optical intensity of the $n$th line, $D$ is the fiber dispersion (in $\mathrm{ps}^{2}$ ), $\Delta \omega$ is the repetition rate of optical frequency comb, $D \Delta \omega$ is the tap delay, $\tau$ is the relative delay between two interferometer arms (which allows for filter tuning), and $\Phi_{n}$ is the phase shift applied to the $n$th comb line with the pulse shaper. Mathematically, Eq. (1) represents a discrete Fourier transform. Then, programmability in the microwave filter's amplitude, $\left|H\left(\omega_{\mathrm{RF}}\right)\right|$, and phase, $\psi\left(\omega_{\mathrm{RF}}\right)=\arg \left[H\left(\omega_{\mathrm{RF}}\right)\right]$, is enabled by proper line-by-line optical processing. In particular, by applying the optical quadratic phase $\left(\Phi_{n}=\right.$ $\beta n^{2}$ ), the filter transfer function will also have a quadratic spectral phase response, i.e., $\psi\left(\omega_{\mathrm{RF}}\right) \sim \psi_{2} \omega_{\mathrm{RF}}^{2} / 2$ The frequency-dependent delay is given by $\tau\left(\omega_{\mathrm{RF}}\right)=$ $-\partial \psi / \partial \omega_{\mathrm{RF}}=-\psi_{2} \omega_{\mathrm{RF}}$, and thus the real-valuated coefficient $\psi_{2}$ establishes the amount of linear dispersion (in $\mathrm{ns} / \mathrm{GHz}$ ) over the designed bandpass. Its value depends both on the comb spectrum and the optical quadratic phase introduced by the shaper (through the $\beta$ parameter). In general, one simply needs to numerically solve Eq. (1) to synthesize the filter transfer function.

Figure 2 shows our optical frequency comb spectrum and the corresponding measured and simulated filter response when $\beta=0.096 \mathrm{rad}$ is programmed with the shaper. This value is chosen to maximize the $\psi_{2}$ parameter, according to a numerical analysis based on Eq. (1). The simulated filter transfer function (dashed curve), obtained from Eq. (1) using the measured comb profile, is in close agreement with that measured (solid curve). The filter gain is $-45 \mathrm{~dB}$, the achieved bandwidth is $\sim 3.7 \mathrm{GHz}$ at $-3 \mathrm{~dB}$, and the center is selected at $3.4 \mathrm{GHz}$ (corresponding to $\tau=32.6 \mathrm{ps}$ tuned with the delay stage).

Next, we explore the phase characteristics of the synthesized electrical filter using a time-domain technique. Figure $\underline{3(a)}$ shows the experimental setup. We
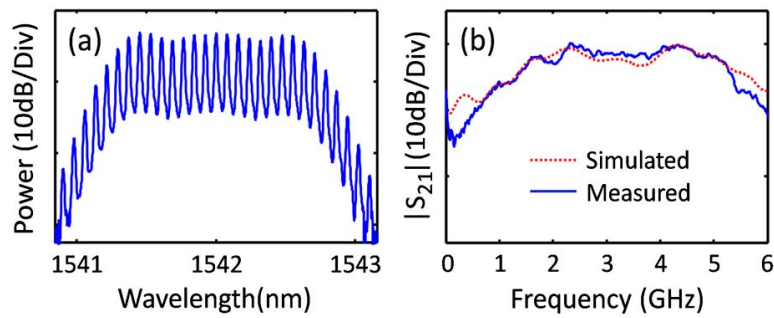

Fig. 2. (Color online) (a) Measured optical spectrum of the flat comb; (b) Corresponding filter response measured (solid curve) and simulated (dashed curve) with the applied coefficients of the quadratic phase to the comb equal to $0.096 \mathrm{rad}$.

send to the MWPF a transform-limited microwave pulse (i.e., a broad-bandwidth pulse with constant spectral phase) with $3.4 \mathrm{GHz}$ center frequency and $-3 \mathrm{~dB}$ bandwidth of $2.8 \mathrm{GHz}$, which repeats periodically every $11 \mathrm{~ns}$. The signal is synthesized with an arbitrary waveform generator operating at $12 \mathrm{GS} / \mathrm{s}$ [see Fig. 3(b)]. The filter modifies the spectral phase of the electrical pulse, leading to an output signal temporally broadened and chirped. The output waveform is amplified, filtered, and finally measured with a real-time sampling scope with $20 \mathrm{GHz}$ analog bandwidth and $50 \mathrm{GS} / \mathrm{s}$ sampling rate. 200 traces are averaged together to enhance the signalto-noise ratio. Figure 3(c) shows the measured signal at the output of the MWPF (left) and its corresponding spectrogram [19] (right) when the programmed phase
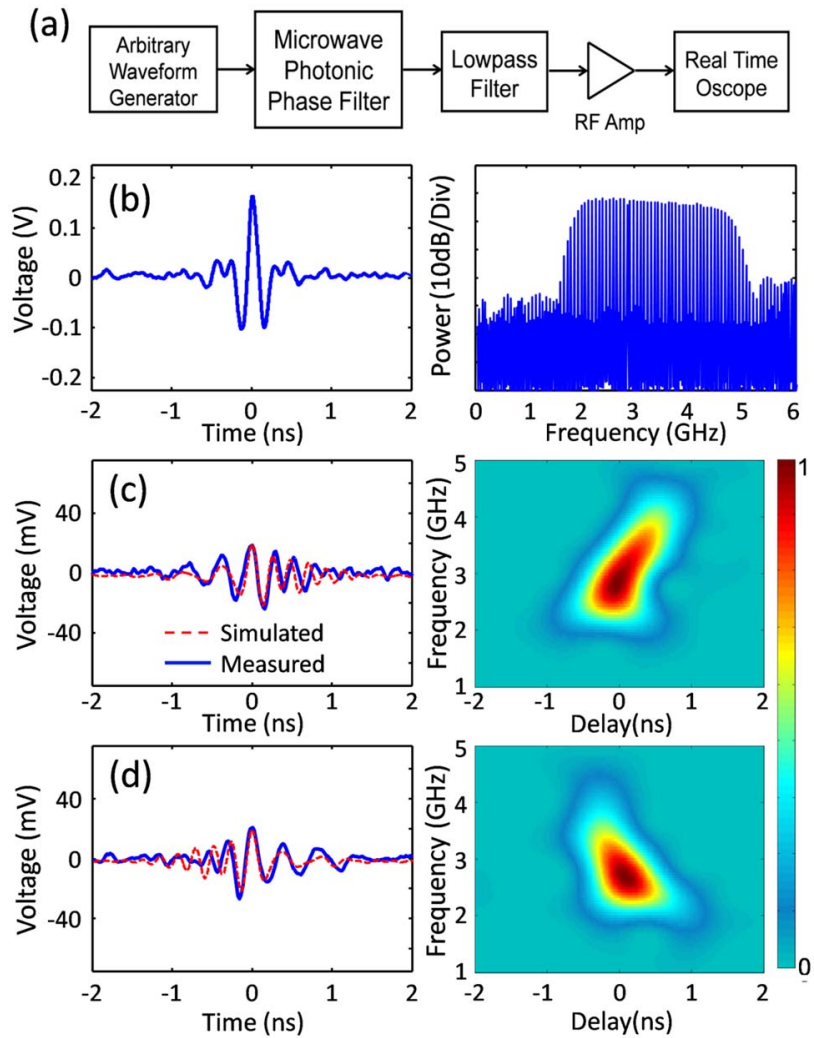

Fig. 3. (Color online) (a) Real-time measurement experimental setup. (b) Measured temporal profile (left) and RF spectrum of the synthesized input pulse. Measured (solid curve) and simulated (dashed curve) output pulse (left) and corresponding spectrogram (right) calculated with a 0.4 ns Gaussian gating function when $\beta$ equals (c) $-0.096 \mathrm{rad}$ and (d) $0.096 \mathrm{rad}$. 

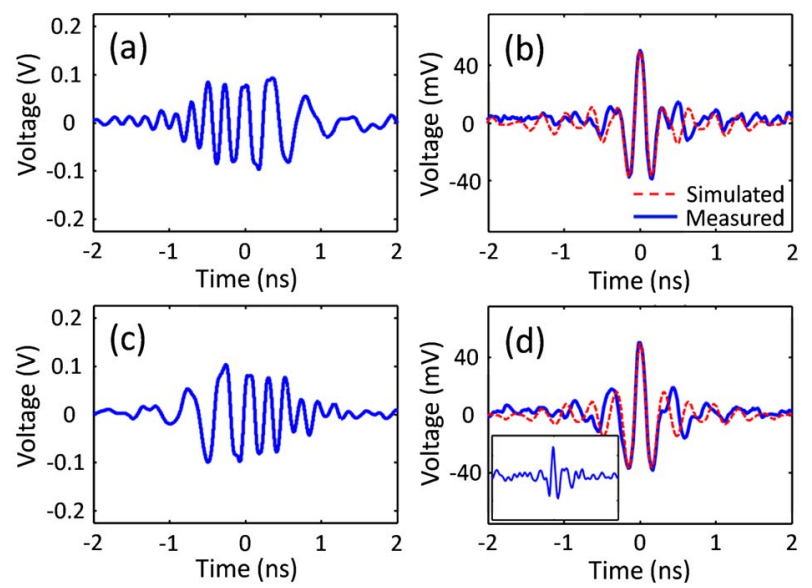

Fig. 4. (Color online) (a), (c) Input linear chirp pulses with $-0.58 \mathrm{~ns} / \mathrm{GHz}$ and $+0.56 \mathrm{~ns} / \mathrm{GHz}$ chirp, respectively, and (b), (d) corresponding measured (solid curve) and simulated (dashed curve) compressed pulses after the matched filter is applied when $\beta$ equals -0.096 and 0.096 , respectively. Inset of (d), single-shot waveform with same $x$ and $y$ axis scale as (d).

coefficient is $\beta=-0.096 \mathrm{rad}$. Through Fourier analysis of the measured electrical signal, the dispersion introduced by the electrical filter is calculated to be $\psi_{2}=$ $+0.6 \mathrm{~ns} / \mathrm{GHz}$, in excellent agreement with the theoretical result calculated using Eq. (1) $(+0.61 \mathrm{~ns} / \mathrm{GHz})$. The slight linear deviation is attributed to small errors in the programmed optical phase. In order to show tuning of the electrical filter's dispersion, we simply reverse the sign of the applied optical phase on the pulse shaper and achieve a dispersion of $-0.56 \mathrm{~ns} / \mathrm{GHz}$, as shown in Fig. 3(d).

These programmable phase filters constitute a convenient platform to implement phase-only matched filtering over nanosecond temporal windows. This operation is commonly used for spectral phase filtering in communication system applications [20]. Such matched filtering cancels the nonlinear spectral phase components of an input signal, resulting in an output waveform compressed to its bandwidth-limited duration. For this aim, we use the scheme in Fig. 3(a). We synthesize an input signal with the same spectral amplitude characteristics as the one in Fig. 3(b) but with a quadratic spectral phase corresponding to a dispersion of either $-0.58 \mathrm{~ns} / \mathrm{GHz}$ [Fig. 4(a)] or $+0.56 \mathrm{~ns} / \mathrm{GHz}$ [Fig. 4(c)]. After sending either of these waveforms to the synthesized microwave photonic phase filters corresponding to Figs. 3(c) and 3(d), respectively, we compensate for the input dispersion and obtain at the output the transform-limited waveforms illustrated in Figs. 4(b) and 4(d). As before, the data are very close to the simulated results, indicating high-fidelity spectral phase control. We also show a typical single-shot waveform in the inset of Fig. 4(d). After $6 \mathrm{GHz}$ low-pass digital filtering, this waveform exhibits an SNR of $\sim 20 \mathrm{~dB}$. Finally, to analyze the fluctuations on a longer temporal scale, we recorded single-shot measurements of a sequence of $\sim 1800$ compressed pulses, one every $11 \mathrm{~ns}$, over a $20 \mu \mathrm{s}$ span. The calculated root-mean-square global phase variation of the compressed pulse is as small as $\sim 0.1 \mathrm{rad}$, with no effect on the amount of dispersion introduced by the MWPF.
In summary, we have demonstrated programmable phase control in a multitap complex-coefficient MWPF. In our interferometric scheme, optical quadratic phase introduced on a $10 \mathrm{GHz}$ frequency comb enables control of filter dispersion in the $\sim \mathrm{ns} / \mathrm{GHz}$ regime. It should be possible to further increase this amount using broader frequency combs. With this platform, we have shown compression of linearly chirped broad microwave pulses with nanosecond temporal apertures to their bandwidthlimited duration. This work opens a new route for linear distortion compensation in large TBWP microwave waveforms, compatible with the demands of modern wireless communication systems.

This project was supported in part by the Naval Postgraduate School under grant N00244-09-1-0068 under the National Security Science and Engineering Faculty Fellowship program. Any opinions, findings, and conclusions or recommendations expressed in this publication are those of the authors and do not necessarily reflect the views of the sponsors. Victor Torres-Company gratefully acknowledges funding from a Marie Curie International Outgoing Fellowship (PIOF-2009-234996).

\section{References}

1. J. P. Yao, J. Lightwave Technol. 27, 314 (2009).

2. J. Capmany, B. Ortega, and D. Pastor, J. Lightwave Technol. 24, 201 (2006)

3. P. Tortoli, F. Guidi, and C. Atzeni, Proc. IEEE Ultrason. Symp. 1, 199 (1994).

4. J. D. McKinney and A. M. Weiner, IEEE Trans. Microw. Theory Tech. 54, 1681 (2006).

5. S. J. Xiao and A. M. Weiner, IEEE Trans. Microw. Theory Tech. 54, 4002 (2006).

6. E. Hamidi and A. M. Weiner, J. Lightwave Technol. 26, 2355 (2008).

7. E. Hamidi and A. M. Weiner, IEEE Trans. Microw. Theory Tech. 57, 890 (2009).

8. Y. T. Dai and J. P. Yao, IEEE Trans. Microw. Theory Tech. 58, 3279 (2010).

9. M. Sagues, A. Loayssa, and J. Capmany, IEEE Photon. Technol. Lett. 19, 1194 (2007).

10. T. Mengual, B. Vidal, and J. Marti, Opt. Commun. 281, 217 (2008).

11. M. Sagues, R. G. Olcina, A. Loayssa, S. Sales, and J. Capmany, Opt. Express 16, 295 (2008).

12. X. Yi, T. X. H. Huang, and R. A. Minasian, IEEE Trans. Microw. Theory Tech. 58, 3088 (2010).

13. E. Hamidi, D. E. Leaird, and A. M. Weiner, IEEE Trans. Microw. Theory Tech. 58, 3269 (2010).

14. M. Song, C. M. Long, R. Wu, D. Seo, D. E. Leaird, and A. M. Weiner, IEEE Photon. Technol. Lett. 23, 1618 (2011).

15. Z. Jiang, C. B. Huang, D. E. Leaird, and A. M. Weiner, Nat. Photon. 1, 463 (2007).

16. M. Bolea, J. Mora, B. Ortega, and J. Capmany, Opt. Express 19, 4566 (2011).

17. M. Li, A. Malacarne, N. Belhadj, S. LaRochelle, J. Yao, and J. Azana, "Flattened optical multicarrier generation of 12.5 GHz spaced 256 channels based on sinusoidal and phase hybrid modulation," presented at the 2011 IEEE Microwave Photonics Conference, Singapore, October 18-21, 2011.

18. M. Fujiwara, J. Kani, H. Suzuki, K. Araya, and M. Teshima, Electron. Lett. 37, 967 (2001).

19. A. M. Weiner, Ultrafast Optics (Wiley, 2009).

20. J. G. Proakis, Digital Communications (McGraw-Hill, 2001). 